\title{
Molecular identification of Candida orthopsilosis isolated from blood culture.
}

\begin{abstract}
The incidence of candidemia and invasive candidiasis have increased markedly due to the increasing number of immunocompromised patients. There are five major medically important species of Candida with their frequency of isolation in the diminishing order namely Candida albicans, Candida parapsilosis, Candida tropicalis, Candida glabrata and Candida krusei. In addition, there are numerous other species of Candida which differ in their genetic makeup, virulence properties, drug susceptibilities and sugar assimilation capabilities. In this report, an unusual Candida species was isolated from the blood of two leukaemic patients. Conventional culture and biochemical tests identified the Candida species as C. parapsilosis. Using fungal-specific oligonucleotide primers ITS1 and ITS4, we managed to amplify the ribosomal RNA gene and its internal transcribed spacer region from the genomic DNA of these isolates. The PCR products were then purified and subjected to automated DNA sequencing using BLAST and CLUSTAL sequence analysis identified these isolates to be Candida orthopsilosis. Candida orthopsilosis is a new species recently identified in 2005, being morphologically indistinguishable from C. parapsilosis and was previously classified as a subspecies of $\mathrm{C}$. parapsilosis. This report highlights the importance of complementing traditional culture and biochemical-based identification methods with DNA-based molecular assays such as PCR as the latter is more superior in terms of its discriminatory power and speed.
\end{abstract}

Keyword: C . orthopsilosis; DNA sequencing; Polymerase chain reaction (PCR). 